\title{
Spike Generator Limits Efficiency of Information Transfer in a Retinal Ganglion Cell
}

\author{
Narender K. Dhingra and Robert G. Smith \\ Department of Neuroscience, University of Pennsylvania, Philadelphia, Pennsylvania 19104-6058
}

The quality of the signal a retinal ganglion cell transmits to the brain is important for preception because it sets the minimum detectable stimulus. The ganglion cell converts graded potentials into a spike train with a selective filter but in the process adds noise. To explore how efficiently information is transferred to spikes, we measured contrast detection threshold and increment threshold from graded potential and spike responses of brisk-transient ganglion cells. Intracellular responses to a spot flashed over the receptive field center of the cell were recorded in an intact mammalian retina maintained in vitro at $37^{\circ} \mathrm{C}$. Thresholds were measured in a single-interval forced-choice procedure with an ideal observer. The graded potential gave a detection threshold of $1.5 \%$ contrast, whereas spikes gave $3.8 \%$. The graded potential also gave increment thresholds approximately twofold lower and carried $\sim 60 \%$ more gray levels. Increment threshold "dipped" below the detection threshold at a low contrast $(<5 \%)$ but increased rapidly at higher contrasts. The magnitude of the "dipper" for both graded potential and spikes could be predicted from a threshold nonlinearity in the responses. Depolarization of the cell by current injection reduced the detection threshold for spikes but also reduced the range of contrasts they can transmit. This suggests that contrast sensitivity and dynamic range are related in an essential trade-off.

Key words: retina; discrimination; contrast; threshold; spike; information

\section{Introduction}

The quality of the visual signal transmitted to the brain is important for perception because it sets the minimum detectable stimulus. As a daylight visual signal is processed by the retina, each layer adds noise (Ashmore and Copenhagen, 1983; Freed et al., 2003) so that the signal quality of a ganglion cell is limited by retinal noise sources (Schellart and Spekreijse, 1973; Reich et al., 1977; Levine and Zimmerman, 1991; Troy and Robson, 1992; Croner et al., 1993; Freed, 2000), implying information loss (Geisler, 1989). The loss is thought to originate partly in selective processing of the signal and partly from noise sources such as stochastic vesicle release and channel gating (Barrett and Stevens, 1972; Schneidman et al., 1998; White et al., 2000; van Rossum et al., 2003). To understand how efficiently information is transferred and how neural mechanisms preserve signal quality, one could measure information loss at each retinal stage. One way is to compare the contrast sensitivity of different stages (Geisler and Davila, 1985; Geisler, 1989; Dhingra et al., 2003). The contrast detection threshold of a ganglion cell, measured in vivo and in vitro, is 2-3\% (Enroth-Cugell and Robson, 1966; Derrington and Lennie, 1982; Linsenmeier et al., 1982; Dhingra et al., 2003), but another stage is required for the comparison.

Received Dec. 3, 2003; revised Feb. 6, 2004; accepted Feb. 9, 2004.

This work was supported by National Institutes of Health Grant MH48168 (R.G.S.). We thank Wilson Geisler and Fred Rieke for their help in developing the ideal observer analysis, and Diego Contreras, Jonathan Demb, Michael Freed, John Robson, and Peter Sterling for helpful discussions.

Correspondence should be addressed to Narender K. Dhingra, University of Pennsylvania School of Medicine, Department of Neuroscience, 123, Anatomy/Chemistry Building, Philadelphia, PA 19104-6058. E-mail: naren@retina.anatomy.upenn.edu.

DOI:10.1523/JNEUROSCI.5346-03.2004

Copyright $\odot 2004$ Society for Neuroscience $\quad 0270-6474 / 04 / 242914-09 \$ 15.00 / 0$
The retinal ganglion cell collects signals from noisy synaptic inputs (Kier et al., 1995; Freed, 2000) into a graded potential, which it converts into a spike train (Diamond and Copenhagen, 1995). The neural mechanism that accomplishes the conversion, the "spike generator," is temporally transient (Sakuranaga et al., 1987; Lankheet et al., 1989; Zaghloul et al., 2003): it selectively filters the signal in the conversion to the spike train. In addition, the coding efficiency of the spike generator is limited by its nonlinear spike threshold: at a subthreshold membrane potential, no spikes are generated (Diamond and Copenhagen, 1995). Furthermore, the stochastically gated ion channels that comprise the spike generator contribute significantly to variability in the spike train (van Rossum et al., 2003). Together, such mechanisms reduce the signal-to-noise $(\mathrm{S} / \mathrm{N})$ ratio (Barlow, 1957), implying that, in the conversion to a spike train, the spike generator removes information, some selectively and some indiscriminately (Juusola and French, 1997).

In some neurons, the information capacity for the spike train is 5- to 15-fold lower than for the graded potential (de Ruyter van Steveninck and Laughlin, 1996; Juusola and French 1997; Haag and Borst, 1998), implying 80-95\% information loss. The loss depends on the temporal structure of the stimulus and the response, on the noise properties of the neuron, and on the time scale used for analysis (Kretzberg et al., 2001). However, it is unknown how efficiently information is transferred from the graded potential to the spike train in a mammalian neuron. To investigate this, we measured contrast discrimination thresholds of guinea pig brisk-transient ganglion cells from graded potential and spike train. Thresholds were measured with an "ideal observer" that analyzed the temporal pattern and variability in the response of the cell (Geisler et al., 1991; Dhingra et al., 2003). We found that the spike train had twofold higher thresholds and 

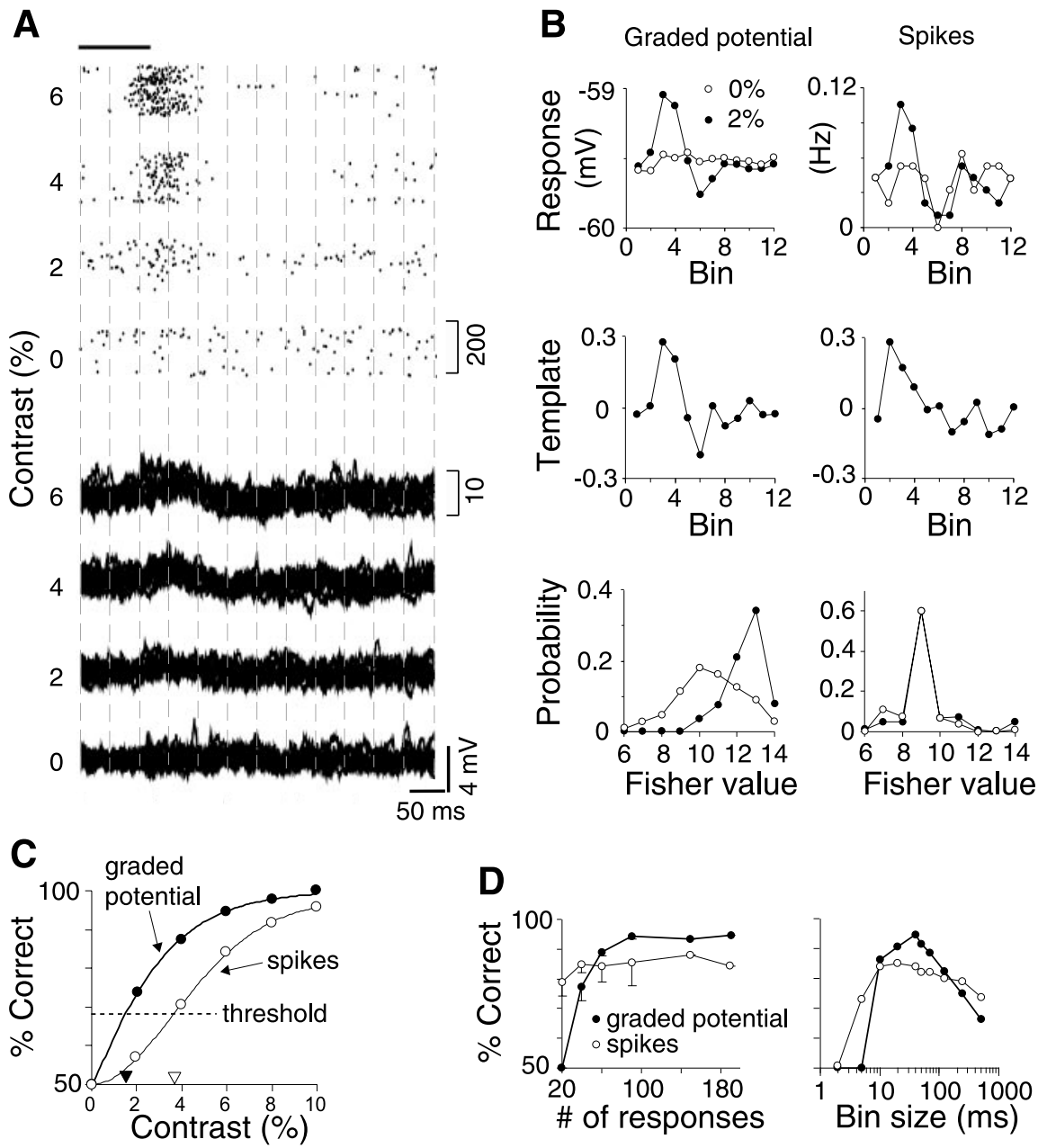

Figure 1. Ideal observer analysis with Fisher LDA. A, Spike (top) and graded potential (bottom) responses of a ganglion cell to a repeated stimulus. Temporal bins are shown by dashed vertical lines. $B$, Average response (top) was computed for each bin for a pair of contrasts ( 0 and $2 \%$ here). The difference between the two averages was then divided by their covariance matrix to generate the optimal template vector (middle). Each response was weighted by the template to produce a Fisher value, taken to generate a PDF for each contrast (bottom). The ideal observer then evaluated the remaining responses in a single-interval forced-choice procedure and decided which contrast most likely evoked a given response. $C$, Graded potential gave higher performance (percentage correct) than spikes. Dashed line shows threshold performance, and arrowheads indicate detection thresholds. Curves are best-fitting cumulative Weibull functions. D, Performance increased with total number of responses used for analysis and maximized at 100 responses (left). A bin size of $30-50$ msec gave maximum performance (right).

carried $40 \%$ fewer gray levels compared with the graded potential response.

\section{Materials and Methods}

Stimulation and recording. These methods have been described in detail previously (Dhingra et al., 2003). Briefly, a retina attached to sclera was removed from an anesthetized adult guinea pig, superfused with Ames medium $\left(4-7 \mathrm{ml} / \mathrm{min}\right.$ at $\left.\sim 37^{\circ} \mathrm{C}\right)$, and visualized through infrareddifferential interference contrast optics. Visual stimuli were generated in Matlab (MathWorks, Natick, MA) (Dhingra et al., 2003), displayed with a 12-bit precision on a color monitor $(640 \times 480$ pixels; $120 \mathrm{~Hz})$, and projected onto photoreceptors. The mean background intensity was $\sim 20,000$ photons $\cdot \mu \mathrm{m}^{2} \cdot \sec$ (equivalent to $\sim 8000 R^{\star} / \mathrm{cone} / \mathrm{sec}$ ) at 535 $\mathrm{nm}$, well into the photopic range. The stimulus was typically a $100 \mathrm{msec}$ square-wave spot repeating at 2 or $4 \mathrm{~Hz}$. Its noise content, measured with a photodiode, was $<0.06 \%$ contrast in the range of $2-70 \mathrm{~Hz}$. Poisson photon fluctuation was also not a concern because it is known to be negligible at photopic backgrounds (Schellart and Spekreijse, 1973; Freed, 2000). This implies that noise in the stimulus did not affect our measurements of performance $(0.5 \%$ or higher contrast, with time bins of $40 \mathrm{msec}$; see below). We chose a spot stimulus with low duty cycle to
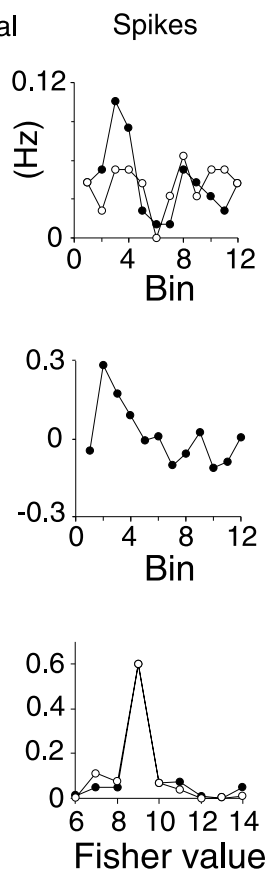

allow better recovery between trials, comparisons with psychophysics that commonly uses this type of stimulus, and analysis of the performance over time. For studying the effect of stimulus frequency on contrast threshold, we used a sine-wave stimulus.

The largest somata (15-25 $\mu \mathrm{m}$ diameter) in the visual streak were targeted for intracellular recording with a sharp electrode containing 1.5 M potassium acetate (tip resistance, 70-200 $\mathrm{M} \Omega$ ). The voltage was recorded at $5 \mathrm{kHz}$, amplified (Neurodata IR-283; Cygnus Technologies, Delaware Water Gap, PA) and digitally stored (Axoscope; Axon Instruments, Foster City, CA). After recording, some cells $(n=7)$ were stained with DiI. Spikes were recognized on the basis of rate of voltage change $(d V / d t)$ exceeding a criterion value. For spike removal, beginning and end time points for linear interpolation were selected on the basis of the spike shape by stepping forward and backward a specified number of time points from the initial rise of the spike. Data between the beginning and end time points was then replaced by a line to approximate the underlying graded potential. We found that the spike removal method was not critical because the spike duration was much smaller than the time bins we used (see below).

One concern with our results from current injection was whether the injected current could have added enough noise to affect the conclusions. The $I-V$ plot was nearly linear (rectification of $<3 \%$ ) in the range of -300 to $300 \mathrm{pA}$, suggesting that the changes in noise were not attributable to changes in electrode resistance (data not illustrated). Electrode noise measured in the bath before and after recording from a neuron was typically $0.1 \mathrm{mV}$ (SD) and remained nearly constant $( \pm 0.02 \mathrm{mV})$ with current over the range of -500 to $500 \mathrm{pA}$, suggesting that electrode noise was not responsible for the noise levels of the measured neuron (typically $1-2 \mathrm{mV}$ ) or their modulation. For the most sensitive measure, noise at low contrast, negative current lessened the noise and positive current increased it (see Fig. 5), consistent with what one would expect for noise originating in voltage-gated channels active near rest $(\mathrm{Na}$, $\left.K_{\mathrm{dr}}\right)$. However, positive current reduced noise at high contrasts, further implying that its direct effect on noise was not the major component, consistent with our conclusions.

Ideal observer analysis. To measure the threshold for contrast detection and discrimination of the ganglion cell, we constructed an ideal observer using Fisher's linear discriminant analysis (Fisher LDA) (Duda and Hart, 1973) (Fig. 1). We chose Fisher's method because it is a template method that projects multi-dimensional data into one dimension to maximize the difference between the two projected response means and minimize the variances and does not assume that responses in neighboring bins are independent. Although Fisher LDA is not guaranteed to be optimal for all possible types of correlation, it is near optimal for data in which the time bin distributions are approximately normal and linearly correlated, the type of correlation expected for noisy temporal signals. On the basis of the temporal pattern of the responses, the ideal observer discriminated between responses to a pair of contrasts in a single-interval forced-choice procedure (Dhingra et al., 2003). The detection task was a special case of discrimination; for detection, one stimulus was zero contrast (mean background), but for discrimination, they were both non-zero contrasts: a higher "test" contrast and a lower "basal" contrast.

Our method differed from traditional LDA in that our discrimination 
between (classification of) the pair of transformed probability distributions used the optimal "likelihood rule" to maximize correct responses and thus did not require any assumptions about normality along the discrimination dimension (Green and Swets, 1988; Geisler et al., 1991). Our method differed from ROC (receiver operating characteristic) analysis in that our discrimination task was a single-interval task in which there were no "false alarms." The likelihood rule (criterion $k=1$; see below) minimized the total error rate instead of parametrizing hits against false alarms as in ROC (Green and Swets, 1988). For measurements of discrimination performance that included only one time bin (see Fig. 6A), the Fisher Template simplified to a constant value, and our method reduced to a pure likelihood rule, which is optimal (Green and Swets, 1988; Geisler et al., 1991).

We recorded the ganglion cell responses to $200-400$ repetitions each of up to 30 contrast values (Fig. $1 \mathrm{~A}$ ). To determine whether the responses to a pair of contrasts were discriminable, we computed the average in 40 msec bins from a randomly selected half of the responses (Fig. $1 B$, top). The difference between the two averages, representing between-class scatter, was divided by the covariance matrix of the binned responses, representing within-class scatter, to generate the optimal template vector (Fig. $1 B$, middle), defined as follows:

$$
\begin{aligned}
\mathbf{T} & =\left(\mathbf{m}_{A}-\mathbf{m}_{B}\right) \cdot \\
& \left(\sum_{i=1}^{n}\left[\left(\mathbf{R}_{i A}-\mathbf{m}_{A}\right)\left(\mathbf{R}_{i A}-\mathbf{m}_{A}\right)^{t}+\left(\mathbf{R}_{i B}-\mathbf{m}_{B}\right)\left(\mathbf{R}_{i B}-\mathbf{m}_{B}\right)^{t}\right]\right)^{-1}
\end{aligned}
$$

where $\mathbf{m}$ is the mean response vector when the stimulus is $A$ or $B, n$ is total number of responses, $\left(\mathbf{R}_{i}-\mathbf{m}\right)$ is deviation of the $i$ th response vector from the mean, and $\left(\mathbf{R}_{i}-\mathbf{m}\right)^{t}$ is transposed $\left(\mathbf{R}_{i}-\mathbf{m}\right)$.

Each response was multiplied bin for bin by the template vector, and the product was summed across all of the bins to give an optimally filtered value (arbitrary units) unique for each response, denoted the Fisher value, given by the following:

$$
F=\sum_{i=1}^{n} t_{i} r_{i}
$$

where $t_{i}$ and $r_{i}$ are the template and response in the $i$ th bin, and $n$ is the total number of bins.

The Fisher values of the responses that generated the template were used to construct for each contrast a probability density function (PDF). A pair of such PDFs represented the ideal observer for a given pair of contrasts (Fig. $1 \mathrm{~B}$, bottom). The ideal observer was then presented the remaining half of the responses (each with its unique Fisher value) and asked to choose for each response which of the two contrasts had most likely been presented. The choice was governed by the likelihood ratio as follows (Green and Swets, 1988):

$$
L=P(F \mid B) / P(F \mid A)
$$

where $F$ is the Fisher value when the stimulus is $B$ or $A$. For $L>k$, the ideal observer chose $B$; for $L<k$, it chose $A$, where $k$ is the criterion. Because the stimuli had equal presentation probability, setting $k=1$ minimized the total error rate (Green and Swets, 1988). When the choice corresponded to the stimulus actually presented, it was "correct." A neurometric function was defined as the fraction of correct responses plotted against the difference between the two contrasts and fitted with a cumulative Weibull function as follows (Quick, 1974) (Fig. 1C):

$$
P=1-0.5 \exp \left(-\frac{c}{\alpha}\right)^{\beta}
$$

where $c$ is the contrast, $\alpha$ is the threshold, and $\beta$ is the slope of the Weibull function. Threshold was defined as the contrast that gave $68 \%$ correct choices (Geisler et al., 1991; Dhingra et al., 2003) (Fig. 1C).

Because the performance measured by the ideal observer may depend on the amount of data collected and the size of temporal bins used in the analysis (Dhingra et al., 2003), we evaluated these parameters. We found that 100 or more responses were required to maximize the performance (Fig. $1 D$, left). As the bin size increased above $\sim 40 \mathrm{msec}$, the performance declined, especially for the graded potential responses (Fig. $1 D$, right), which is consistent with our previous finding that ganglion cell responses have more information in their temporal pattern (Dhingra et al., 2003). At bin sizes less than $\sim 10 \mathrm{msec}$, the performance also declined because the data were insufficient to give smooth PDF histograms. In the previous study using larger data sets (800 trials), we found that performance plateaued at bin sizes shorter than $40 \mathrm{msec}$ (Dhingra et al., 2003), which was consistent with the relatively slow responses recorded near threshold contrast.

To measure discrimination performance over the entire contrast range, we defined "increment threshold" as the just detectable contrast increment above the basal contrast, computed as the difference between the discrimination threshold and the basal contrast. We fitted this with the following function (Boynton et al., 1999):

$$
R(C+\Delta C)-R(C)=\Delta R
$$

where $R(C+\Delta C)$ and $R(C)$ are the response of the cell to the test and the basal contrast, respectively, $\Delta C$ is the predicted increment threshold, and $\Delta R$ is the noise in the response at threshold. Equation 5 was rearranged to derive $\Delta C$ as follows:

$$
\Delta C=\hat{C}[\Delta R+R(C)]-C
$$

where $\hat{C}$ is inverse response function or the contrast that produces a given response. Equation 6 solved in conjunction with the contrast response function (Eq. 9; see below) gave $\Delta C$ (see Fig. $4 A$, solid lines).

Signal, noise, and contrast response function. For the purpose of modeling the increment threshold (see Fig. 4A) (see above), the signal was defined as the mean response in a $10 \mathrm{msec}$ bin, averaged over all of the trials, as follows:

$$
\text { Signal }=\left(\sum_{i=j=1}^{i=m, j=n} r_{i j}\right) / m \cdot n
$$

where $r_{i j}$ is the light evoked response in the $i$ th trial and $j$ th bin, and $m$ and $n$ are the numbers of trials and bins in a trial, respectively. Similarly, the noise was defined as follows:

$$
\text { Noise }=\left(\sqrt{\sum_{j=1}^{n} \sigma_{j}^{2}}\right) / n
$$

where $\sigma_{j}$ is the $\mathrm{SD}$ of the response in the $j$ th bin.

The contrast response function of a cell was fitted with a modified Naka-Rushton equation (Naka and Rushton, 1966; Boynton et al., 1999), as follows:

$$
R(C)=R_{\max } \cdot \frac{C^{p+q}}{C^{q}+\sigma^{q}}
$$

where $R(C)$ is cell response at contrast $C, R_{\max }$ is maximum response, $\sigma$ is half-saturation constant, and $p$ and $q$ are parameters that determine the shape of the function at high and low contrasts, respectively.

\section{Results}

The brisk-transient ganglion cells reported here $(n=40)$ resembled in morphology and physiology the "brisk-transient" $/ \mathrm{Y} / \alpha$ cell in cat and rabbit (Demb et al., 2001; Dhingra et al., 2003; Zaghloul et al., 2003). The cells were mostly OFF-center type, partly because, in our preparation, they are approximately threefold more frequently encountered than ON type, and partly because $\mathrm{ON}$ cells with higher maintained spike rates were difficult to hold for long periods (Dhingra et al., 2003). The cells had monostratified, radiating dendrites (400-700 $\mu \mathrm{m}$ diameter) (Fig. 2A). The input resistance at rest was $20-70 \mathrm{M} \Omega$. The resting 
A
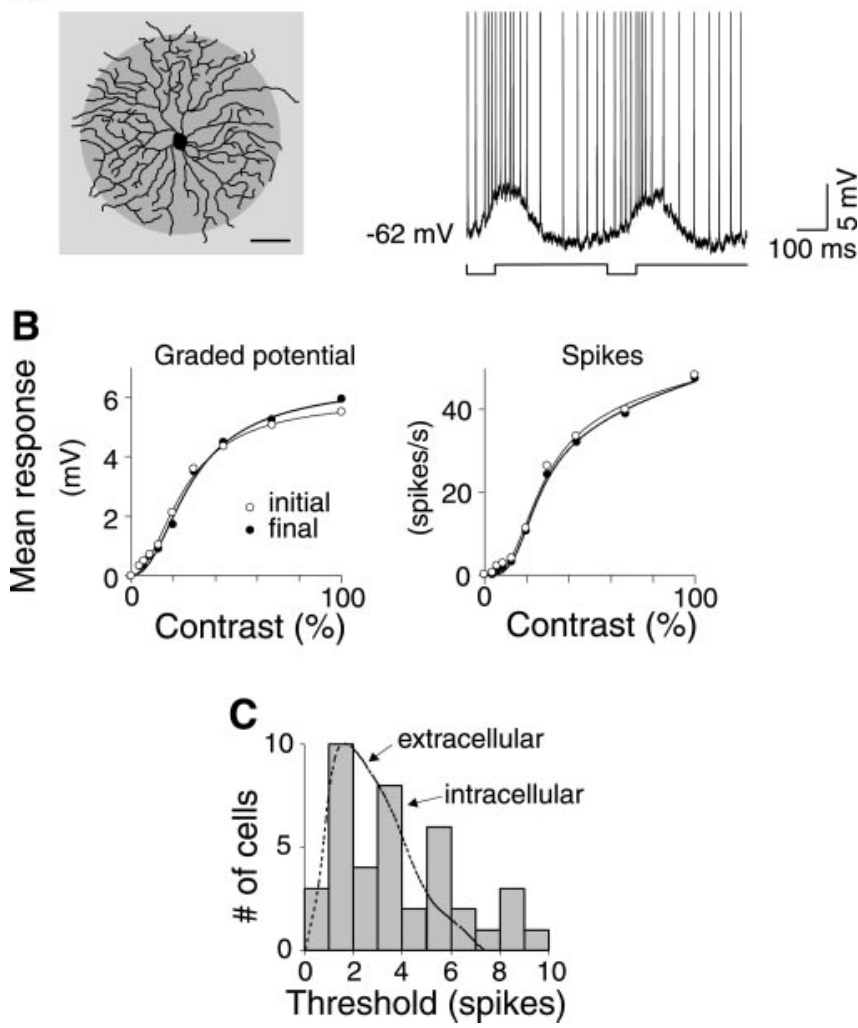

Figure 2. Brisk-transient ganglion cells gave stable responses in vitro. A, Left, A brisktransient OFF-center cell from visual streak, stained with Dil. Dark spot represents stimulus that matched the receptive field center of the cell. Scale bar, $100 \mu \mathrm{m}$. Right, Intracellular response of the cell (stimulus, $100 \mathrm{msec}, 10 \%$ contrast, $2 \mathrm{~Hz}$ ), showing spikes (truncated) riding over graded potential response. $B$, Contrast response function of the cell measured before (initial) and after (final) recording the main data were same, implying stationarity. Curves are best nonlinear fits (Eq. 9). C, Detection thresholds for spikes recorded intracellularly were similar to thresholds for spikes recorded extracellularly (dotted line) (Dhingra et al., 2003).

membrane potential was $-61.7 \pm 5.3 \mathrm{mV}$ (mean $\pm \mathrm{SD}$ ) as reported previously (Demb et al., 2001; Zaghloul et al., 2003).

The responses typically peaked at $100 \mathrm{msec}$ (range of 70-150 msec) after the stimulus onset (Figs. 1A, 2A). The maintained spike rate of the OFF cells was relatively low $(6.6 \pm 7.3 \mathrm{~Hz}$; mean $\pm \mathrm{SD}$ ) because of the high background intensity, as reported previously for in vivo recordings in cat (Cleland et al., 1973; Troy and Robson 1992) and in vitro recordings in guinea pig (Dhingra et al., 2003; Zaghloul et al., 2003). The contrast response function of a cell measured at the beginning and end of a recording that lasted for up to $3 \mathrm{hr}$ were similar, implying stationarity (Fig. 2B). The detection thresholds for the spikes recorded here intracellularly were compared with the thresholds for spikes recorded previously extracellularly (Dhingra et al., 2003). The two sets of cells were selected with the same criteria and used the same stimulus. They were indistinguishable in their soma size, dendritic morphology, receptive field size, maintained rate, frequency tuning, and contrast response. Their thresholds were also similar $(p>0.05 ; \mathrm{df}=73$; paired $t$ test) (Fig. $2 C$ ), suggesting that the cells reported here were not significantly damaged by the sharp electrode. Furthermore, to understand the effect of a small current leak that might have existed, we tested performance in a subset (nine) of the cells (see below, Current injection).
A

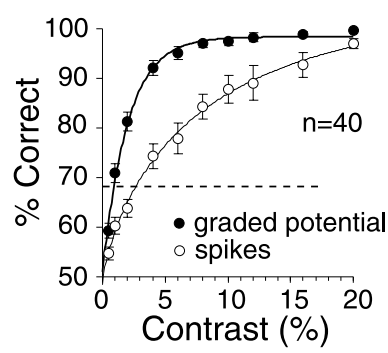

C

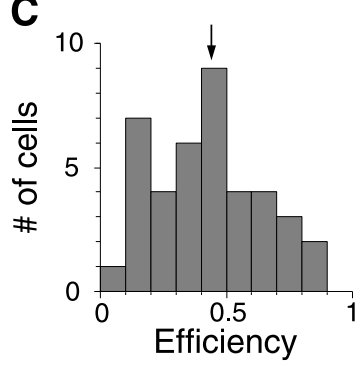

E

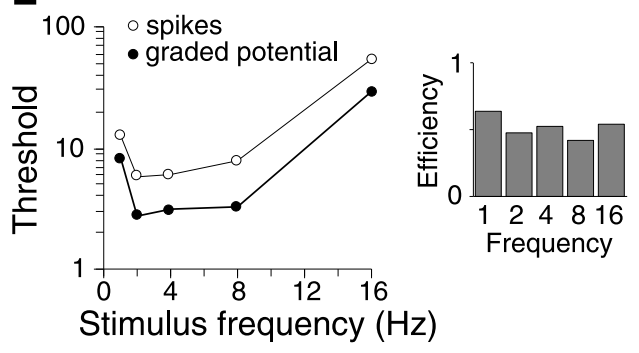

Figure 3. Spikes have twofold higher detection thresholds than graded potential. A, Contrast detection performance (percentage correct; mean $\pm \mathrm{SE}$ ) for graded potential response was consistently higher than for spikes. $B$, Detection thresholds for spikes were always higher than for graded potential. Solid line represents a case in which spikes and graded potential would produce equal thresholds. C, Efficiency of spike generator (mean of $\sim 0.5$; arrow) measured as ratio of detection thresholds (graded potential/spikes) across population. D, Efficiency was mildly correlated with the maintained spike rate of the cell but was always less than one. $E$, Detection threshold of a representative cell for both graded potential and spikes changed with stimulus frequency, but efficiency (right) was relatively constant.

\section{Contrast detection}

First, we estimated the detection threshold from the contrast response function of the cell (Figs. $1 A, 2 B$ ) and then measured the threshold from the graded potential and spike responses. At each contrast, the graded potential gave more correct choices than the spikes (Fig. $3 A$ ), invariably producing a lower detection threshold. The threshold for the graded potential $(1.47 \pm 0.22$; mean \pm SE; $n=40$ ) was $\sim 60 \%$ lower than for spikes $(3.79 \pm 0.4 ; p \ll$ 0.0001 ; df $=39$; paired $t$ test) (Fig. $3 B$ ). Efficiency of the spike generator of the cell measured as the ratio of the detection thresholds (graded potential/spikes) was $0.42 \pm 0.03$ (Fig. $3 C$ ). This efficiency was mildly correlated (correlation coefficient of 0.48 ) with the maintained spike rate of the cell but was always less than one (Fig. 3C,D).

There could be several possible explanations for the low efficiency of the spike generator. First, because the spike generator passes high stimulus frequencies (Lankheet et al., 1989), it seemed conceivable that the low efficiency was attributable to the relatively low stimulus frequency ( 2 or $4 \mathrm{~Hz}$ ). To test this, we repeated the above measurements at frequencies up to $16 \mathrm{~Hz}$ and found $(n=2)$ that, although the thresholds for graded potential 
A
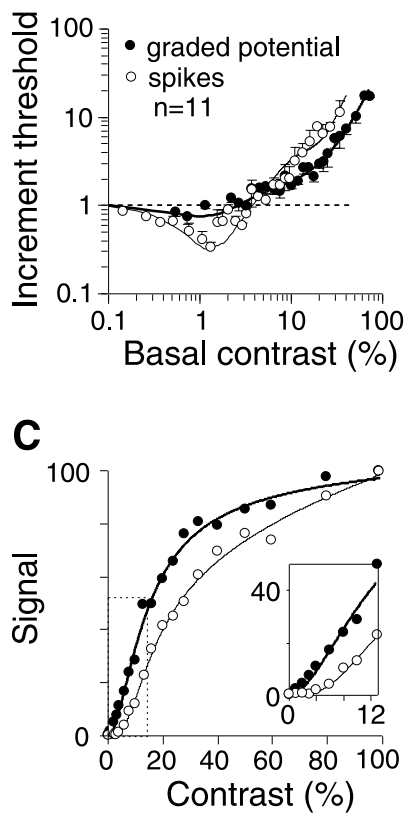

B
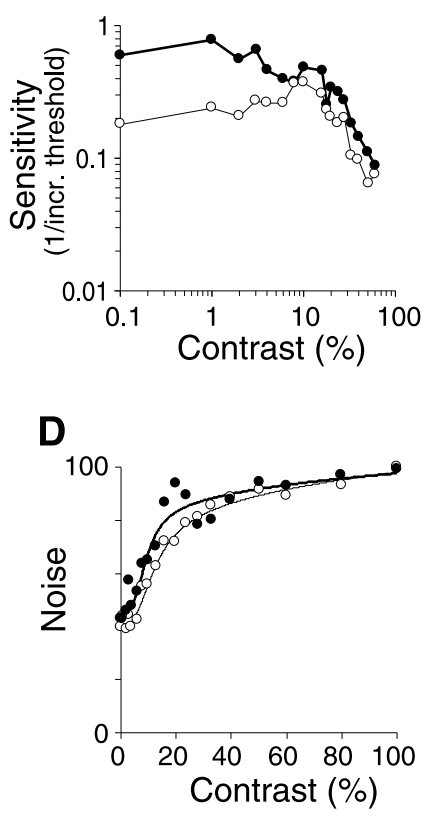

Figure 4. Spikes have higher discrimination thresholds than graded potential. $A$, Increment thresholds (mean \pm SE) were low (high sensitivity) at low contrasts $(<20 \%)$ but increased rapidly at higher contrasts. Because detection thresholds varied among cells (graded potential, $1.7 \pm 0.9 ;$ spikes, $5.6 \pm 3.9$; mean \pm SD), we normalized the increment threshold of each cell to its detection threshold (dashed line). At very low contrasts, the increment threshold "dipped" below detection threshold, more prominently for spikes. Curves are derived from the contrast response signal and noise functions (Eqs. 5-9). Symbols and number of cells also apply to other panels. $B$, Contrast sensitivity, measured as inverse of the increment threshold, remained relatively high up to $\sim 20 \%$ basal contrast but declined rapidly at higher contrasts. C, Evoked response (mean signal, normalized; Eq. 7) showed threshold nonlinearity ( $<5 \%$ contrast; inset), rose rapidly up to $\sim 20 \%$ contrast, and then saturated. Area in the dotted box is magnified in the inset. Graded potential response threshold was lower (1-2\%) than spike response threshold (3-4\%) (inset). Curves are best nonlinear fits. D, Mean noise (normalized; Eq. 8) was relatively high at low contrasts, increased with up to $\sim 20 \%$ contrast, and saturated at higher contrasts.

and spikes both changed with stimulus frequency, their ratio remained relatively constant (Fig. $3 E$ ). Second, it seemed possible that a simple spot in the receptive field center is not the optimal stimulus for the spike generator. To test this, we measured the spatiotemporal receptive field profile of the cell $(n=2)$ using a white-noise stimulus and a reverse-correlation analysis (Jones and Palmer, 1987) and presented the spatiotemporal profile as the stimulus. Because the reverse correlation gives the average stimulus kernel, when measured near threshold, this stimulus is near optimal. We found that, for these two cells, the ratio of detection thresholds (graded potential/spikes) produced by the optimal stimulus $(0.54 \pm 0.17)$ was similar to that produced by the simple spot $(0.55 \pm 0.19)$.

\section{Contrast discrimination}

The increment threshold, for both the graded potential and spikes, initially declined with the basal contrast (Fig. 4A). As the contrast increased further, the increment threshold rose, producing a "dipper effect" (Nachmias and Sansbury, 1974). The "dip" for the graded potential was shallower $(\sim 25 \%$ below detection threshold) than for the spikes $(\sim 65 \%)$. The basal contrast that gave the lowest increment threshold matched the detection threshold of the cell. The ratio of increment thresholds (graded potential/spikes) was as low as 0.3 below $\sim 20 \%$ basal contrast but

increased at higher contrasts, producing a higher mean $[0.66 \pm$ 0.04 (SE)] than for detection. Below 20\% contrast, the increment threshold remained relatively low and close to the detection threshold, implying high sensitivity, but at higher contrasts they increased rapidly, implying much lower sensitivity (Fig. 4A).

To characterize the precision of contrast carried by the graded potential and spike responses, we computed the cumulative sensitivity or number of "gray levels," defined as the inverse of the increment threshold (Fig. 4B) (Barlow et al., 1987) integrated over contrast. Because the increment threshold rose rapidly at high basal contrasts (above 20\%), they did not add many extra gray levels. The reason is that, at these contrasts, the ganglion cell responses saturated but the noise did not decrease (Fig. 4C,D), requiring a greater contrast difference to discriminate a contrast pair. For both the graded potential and spikes, the signal (Eq. 7) had nonlinearities at very low contrasts, at which the cells did not respond much, and at high contrasts, at which the response saturated (Fig. 4C). The noise amplitude (Eq. 8) showed similar nonlinearities (Fig. 4D). We predicted the increment threshold on the basis of these signal and noise functions and found that it matched the measured increment threshold closely (Fig. 4A, curves vs symbols).

The number of gray levels, measured by integrating the sensitivity over contrast (Fig. $4 B$ ), was 15.5 for the graded potential and 10.1 for the spikes, which could not have been predicted on the basis of the detection thresholds alone. Thus, for the discrimination task used here, the graded potential of the ganglion cell represents the stimulus with a resolution of $\sim 4$ bits and the spike train with $\sim 3$ bits. We also measured the dynamic range of the contrasts that are encoded linearly in the responses by computing the contrast at which the response was half-maximum (same as $\sigma$ in Eq. 9). The "half-max" for the spikes $(23.9 \pm 2.3$; mean \pm SE) was significantly higher than for the graded potential response $(17.5 \pm 1.8 ; p<0.05 ; \mathrm{df}=10$; paired $t$ test $)$.

\section{Current injection}

We hypothesized that one reason for the higher contrast detection and discrimination thresholds for spikes is that the cell rests at a potential negative to the threshold for spiking. Thus, a very small synaptic input that drives the graded potential response may not raise the resting potential enough for the cell to spike. If so, depolarizing the cell by injecting positive current might induce more spiking and thus reduce the detection and discrimination thresholds, whereas hyperpolarizing the cell would have the opposite effect.

We found that depolarizing the cell increased the spike response but slightly decreased the graded potential response, and hyperpolarizing had the opposite effect (Fig. 5A). The increase in the spike response was likely attributable to a shift in the resting potential with respect to the spike threshold, whereas the decrease in the graded potential response was probably attributable to the "clamping effect" of the spike-generating currents on the membrane potential (Diamond and Copenhagen, 1995), in which depolarization above spike threshold is limited by the $\mathrm{K}^{+}$currents during spike recovery. We quantified these changes by measuring signal and noise over the entire contrast range when the cell was depolarized, at rest, or hyperpolarized (Fig. 5B). In addition to the changes in the signal described above, there were considerable changes in the noise amplitude. Depolarization increased the noise in both graded potential and spikes at low contrasts but slightly decreased noise at high contrasts, which for the spike response resulted in a higher $\mathrm{S} / \mathrm{N}$ ratio (Fig. $5 B$, bottom).

The changes in signal and noise affected the performance of 
A
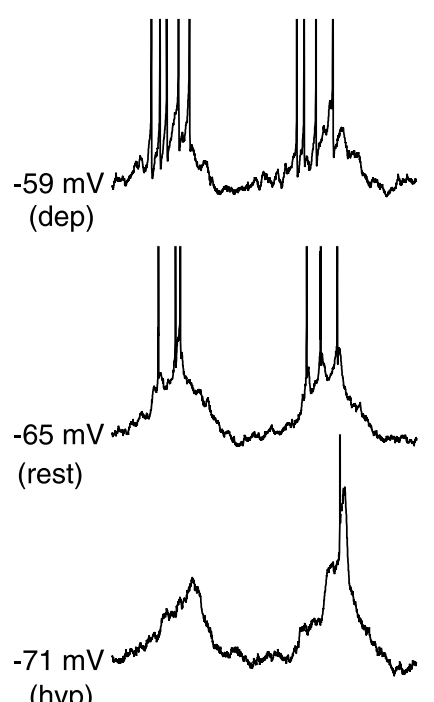

(hyp)

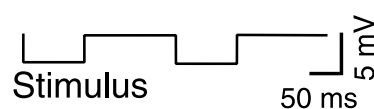

B
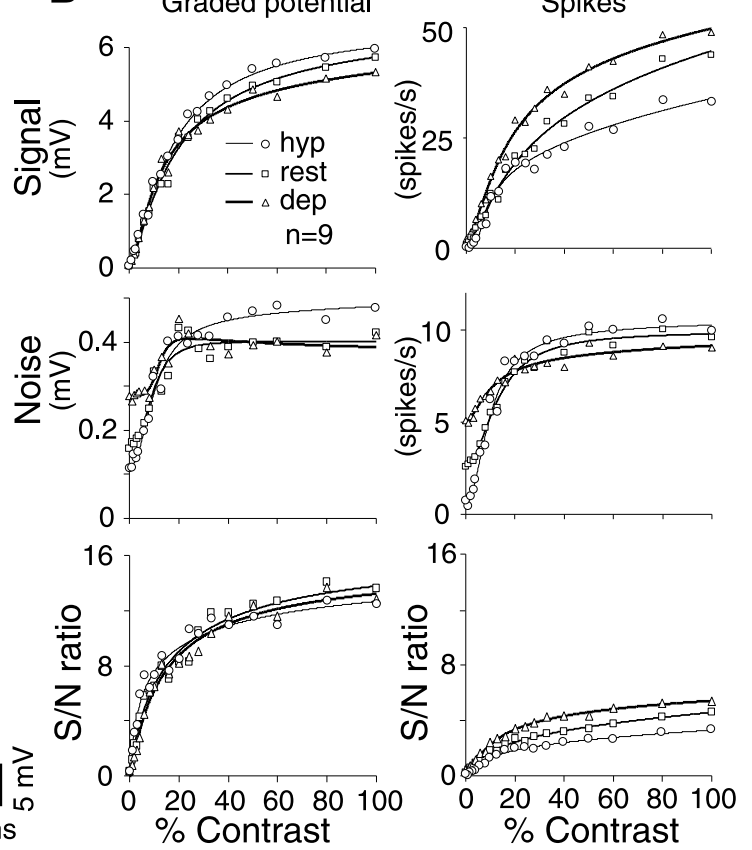

Figure 5. Current injection induced changes in signal and noise. A, Intracellular responses of a representative cell, depolarized (dep), at rest (rest), or hyperpolarized (hyp) by current injection. Depolarizing current increased spike response but slightly decreased graded potential response. Hyperpolarizing had the opposite effect. $B$, Depolarizing current increased the $S / \mathrm{N}$ ratio for spikes attributable to higher signal and lower noise over the entire contrast range but affected the $S / N$ ratio for graded potential less because, in this case, extra spikes clamped the membrane potential.

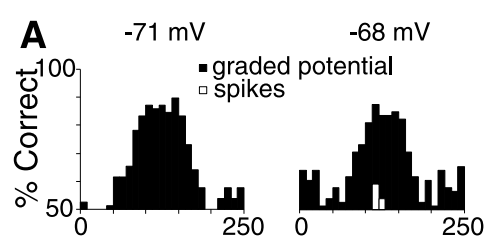

B

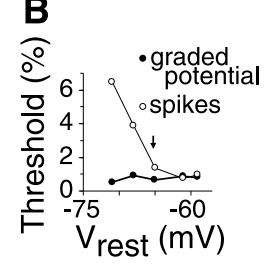

C

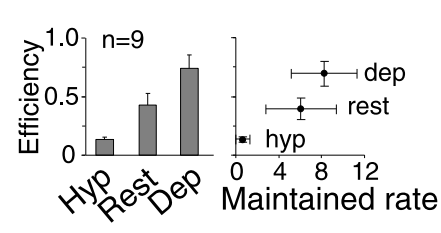

$-65 \mathrm{mV}$
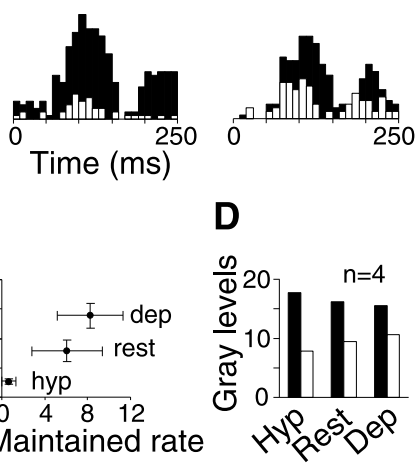

$-59 \mathrm{mV}$
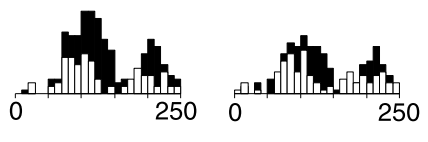

E

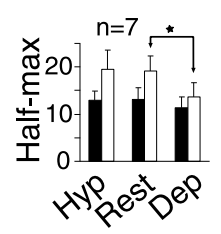

Figure 6. Depolarization caused higher efficiency of the spike generator but lower dynamic range. $A$, The performance of the ganglion cell varied over time, with contributions from depolarization at stimulus on (first peak) and hyperpolarization at stimulus off (second peak). The two peaks reflect times during the biphasic response at which the signal was significantly greater than noise. Each bar represents optimal performance (in detecting $2 \%$ contrast) for one time bin (see Materials and Methods). When the cell (same as in Fig. 5A) was depolarized in steps (left to right panels), performance for graded potential decreased, whereas that for spikes increased. Values at the top are the resting potential. B, Detection threshold (same cell as in Figs. 5A, 6A) for spikes declined, but threshold for graded potential increased slightly with depolarization. Note that the lowest threshold for spikes (at $-60 \mathrm{mV}$ ) is slightly higher than the lowest threshold for graded potential (at $-71 \mathrm{mV}$ ), implying an efficiency of less than one. Arrow points to resting potential with no current injection. C, Left, Efficiency (mean \pm SE; same cells as in Fig. 5B), measured as ratio of thresholds (graded potential/spikes), increased during depolarization. Efficiency for depolarized state (dep) may be slightly overestimated because of clamping effect (see Results). Right, Efficiency was correlated with maintained rate. Horizontal and vertical bars are SEs of maintained rate and efficiency, respectively. $D$, Number of gray levels, calculated as inverse increment threshold integrated over contrast (see Results) (Fig. $4 B$ ), decreased slightly during depolarization for graded potential (black bars) but increased for spikes (white bars). E, Dynamic range, measured as half-max contrast, did not change much for graded potential (black bars) when cells were hyperpolarized or depolarized. Half-max for spikes (white bars) also did not change much when cells were hyperpolarized but decreased significantly $\left({ }^{*} p<0.05\right)$ when cells were depolarized. the ideal observer. To determine how this affected different parts of the response, we measured performance for individual temporal bins (Fig. 6A). Because this performance was calculated from single bins using the likelihood rule that did not require the Fisher template (see Materials and Methods), it was an optimal calculation. When the cell was depolarized, the performance for the graded potential response declined, possibly attributable to the clamping effect of the extra spikes (Fig. $6 A)$. Because depolarization raised the resting potential closer to the reversal potential for the graded potential response $(-40 \mathrm{mV}$; data not illustrated) (Demb et al., 2001; Zaghloul et al., 2003), the performance decreased in the early bins (Fig. $6 \mathrm{~A}$, first peak) but increased in the later bins (second peak). The performance for the spike response increased on depolarization, in both initial and later parts of the response. Depolarization led to higher maintained spike rate so that both $\mathrm{ON}$ and OFF components of the response contained information (Fig. 6A).

Changes in the individual bin performance were reflected in the detection and discrimination thresholds we measured with Fisher LDA. On depolarization, the detection threshold for the graded potential increased slightly, whereas that for the spike response decreased (Fig. $6 B$ ), resulting in increased efficiency (Fig. 6C). The efficiency for detection, measured at rest to be $0.43 \pm 0.1$ (mean \pm SE; $n=9$ ), increased on depolarization to $0.74 \pm 0.11$ and decreased on hyperpolarization to $0.13 \pm 0.02$ (Fig. 6C). Similarly, the efficiency for discrimination, measured at rest to be $0.57 \pm 0.05(n=4)$, increased on depolarization to $0.73 \pm 0.09$ and decreased on hyperpolarization to $0.45 \pm$ 0.07 (data not illustrated). The change in efficiency, as expected, was correlated with the maintained spike rate of the cell (Fig. 6C).

Current injection also slightly changed the number of gray levels in the responses. On depolarization, the gray levels in the graded potential decreased, but in the spike response increased (Fig. 6D). However, for spikes, the value of the half-max contrast, which at rest was $19.2 \pm 3.2$ (mean \pm SE; $n=7$ ), decreased significantly $(13.7 \pm 3 ; p<0.05 ; \mathrm{df}=6$; paired $t$ test) (Fig. $6 E$ ) when the cell was depolarized, because the spike response now rose with contrast more rapidly and saturated at a relatively lower contrast (Fig. 5B).

\section{Discussion}

We measured the contrast detection and discrimination thresholds of the brisk- 
transient ganglion cell of mammalian retina. The graded potential had approximately twofold lower thresholds and 60\% more gray levels than the spike train (Figs. 3, 4), implying that, in the process of generating spikes, $\sim 0.7$ bit of information about the visual discrimination is lost. Previous reports have estimated a discrimination ratio (photoreceptors/behavior) somewhere between 4 and 10, equivalent to an information loss of 2-3 bits (Banks et al., 1987, 1991; Davila and Geisler, 1991). This might seem surprising, because without an important design principle to suggest such a loss, it is often assumed that sensory systems are near optimal (Barlow, 1957; Shapley and Victor, 1986; Bialek and Owen, 1990). However, if each stage in the visual pathway presents a decrement similar to our finding, an overall loss of 2-3 bits is plausible.

The ideal observer method used in our previous study (Dhingra et al., 2003) assumed that responses in neighboring time bins were independent. Although this assumption seemed reasonable for spikes, it was inappropriate for the graded potential, which showed correlations over $15 \mathrm{msec}$ (data not illustrated). The ideal observer used here, Fisher LDA, is a template method that does not assume independence between bins (see Materials and Methods) and is thus more appropriate. To check this method, in an alternate computation, we used optimal likelihood discrimination analysis for single bins without Fisher LDA (Fig. 6A) and, over most of the time bins, found an efficiency factor of $\sim 0.5$ (at rest), similar to that computed with Fisher LDA (Figs. 3, 4) and S/N ratio (Fig. 5).

\section{Threshold nonlinearity}

Our measurements showed that the ganglion cell did not respond well to very low contrasts, as observed previously (Chichilnisky and Kalmar, 2002; Dhingra et al., 2003; Zaghloul et al., 2003). We found that this threshold nonlinearity could predict "dippers" that matched the depth of those we found (Fig. $4 A$ ), as suggested in other reports from retina, cortex, and behavior (Nachmias and Sansbury, 1974; Barlow et al., 1987; Dhingra et al., 2003). One source for the threshold nonlinearity in the spike responses could be the spike threshold, because a ganglion cell whose resting potential is below threshold would require a larger contrast to produce a spike (Diamond and Copenhagen, 1995). However, because the threshold nonlinearity also existed in the graded potential responses (Fig. 4B) (Zaghloul et al., 2003), it may also originate in the nonlinear release of neurotransmitter from bipolar cells (Demb et al., 2001). The dipper effect is thus a consequence of the threshold nonlinearity and indicates processing by retinal circuitry.

\section{Resting potential and effect of current injection}

The threshold nonlinearity in the response of a ganglion cell, as mentioned above, might seem suboptimal because it reduces the sensitivity to fine contrasts near zero. If this nonlinearity is attributable to the threshold for spiking, raising the resting potential of the cell by a few millivolts to increase the maintained rate might reduce the nonlinearity and thus improve the sensitivity to low contrasts. Indeed, we found that depolarizing the cell increased the sensitivity of its spike train to low contrasts, resulting in higher efficiency of the spike generator (Figs. 5, 6). However, this also caused the light response to saturate at a lower contrast, thus reducing the range of contrasts the ganglion cell could reliably transmit (Fig. $6 E$ ). In addition, it is known that a high maintained rate tends to reduce latency of the first spike in response to a stimulus but also increases the variability of the first spike (van Rossum et al., 2003). Therefore, the effect of regulating the rest- ing potential is apparently a balance between response latency, precision in spike timing, sensitivity to low contrasts, and discriminability at high contrasts.

The resting potential of a ganglion cell is a summation of all of the synaptic inputs and other membrane currents that comprise the spike generator (Lipton and Tauck, 1987; Ishida, 1995; Taylor et al., 1996; Fohlmeister and Miller, 1997; Wang et al., 1998; Benison et al., 2001; van Rossum et al., 2003). That the energy supply of the retina to support these currents is limited (Laughlin et al., 1998) implies an intrinsic requirement to regulate the resting potential and spike rate of the cell. Adaptation to reduce the spike rate originates at least in part from inhibitory currents and inactivation of $\mathrm{Na}^{+}$currents in the membrane of the ganglion cell, implying active control of spiking properties (Fohlmeister and Miller, 1997; Benison et al., 2001; Kim and Rieke, 2003; van Rossum et al., 2003). Feedback to amacrine cells through gap junctions (Vaney, 1991; Dacey and Brace, 1992) might also be a source for adaptation of the spike rate. Because the resting potential is so important for the processing of information into the spike train, it seems likely that the resting potential is controlled by a feedback mechanism that dynamically regulates the coding strategy of the ganglion cell. The threshold nonlinearity, because it is a consequence of the spike threshold, may be part of such a strategy.

\section{Sensitivity versus dynamic range}

The ability to discriminate fine increments of contrast is important because it limits what can be seen under difficult conditions, e.g., fast motion or camouflage. This limitation is reflected in our measurement of the number of distinct gray levels a ganglion cell can transmit (Fig. 4E). Although the detection threshold for the spike train was $\sim 3 \%$, which suggests that the ganglion cell could partition a visual scene into 30 gray levels, we found that the increment threshold rose dramatically (to $20 \%$ or more) at higher contrasts, resulting in a total of only 10 gray levels. Because the number of spikes in response to our stimulus was limited to $\sim 20$ at very high contrast (data not illustrated), apparently the spike generator codes each gray level with one to two extra spikes. However, the representation of gray levels by spikes was not uniformly distributed over contrast (Fig. $4 B$ ), because, at very low or high contrasts, response nonlinearities (threshold nonlinearity and saturation) and variability in spike timing reduce the information available in each extra spike (Berry et al., 1997). This suggests a trade-off between contrast sensitivity and dynamic range of a ganglion cell in the shape of its response function: because of a limited dynamic range, the ganglion cell sets low sensitivity to high contrasts to preserve high sensitivity to low contrasts. This trade-off is regulated by the resting potential, especially in ganglion cells with a pronounced threshold nonlinearity.

The graded potential response was also limited $(\sim 15$ gray levels), as reported previously for an insect neuron (Simmons, 1999), suggesting that the dwindling number of gray levels in the ganglion cell spike response is not caused entirely by a limitation inherent to spike coding. Rather, the trade-off between sensitivity and dynamic range is a coding strategy that allows precise responses to some visual stimuli and benefits graded potential and spike train alike. The reason the spike response gave lower sensitivity than the graded potential at high contrasts (Fig. 4D) is that the spike response shows the effect of additional noise sources, including stochastic voltage-gated channels (van Rossum et al., 2003). Therefore, the difference between the number of gray levels in the graded potential and spike responses originates in at 
least two mechanisms, the threshold for spiking and voltagegated channel noise.

\section{Implications for visual processing}

In a system comprising several gain stages, the relationship between signal and noise can provide clues about the loci for generating gain and noise. Noise mixed with the visual signal would drop when passed through a gain-reduction mechanism, such as static saturation. Because at higher contrasts the signal saturated but this did not reduce the noise (Figs. 4, 5), the source of noise must be more proximal than the saturation mechanism. For example, if the saturation in the graded potential is generated in the calcium-activated vesicle release mechanism in bipolar cells, our finding of robust noise in the ganglion cell at high contrasts implies that the noise must originate in the ganglion cell, either from the bipolar or other synaptic inputs (Freed, 2000) or in the spike generator (Schellart and Spekreijse, 1973; van Rossum et al., 2003).

Our finding that the contrast threshold for the spike train is twofold higher than that of the graded potential raises several points about visual processing. First, the regulation we found of the dynamic range and sensitivity of the spike train by its maintained rate suggests that active control of resting potential is important (van Rossum et al., 2002, 2003). Second, because of the importance of absolute contrast threshold for the brain, one wonders whether the information loss by the spike generator represents another essential trade-off. Although a hybrid analogto-digital form of processing has advantages for the brain (Sarpeshkar and O'Halloran, 2002), some information loss is expected in a selective analog-to-digital conversion process, such as the spike generator. It has also been suggested that noise in the spike train may have utility to prevent synchronization of parallel visual pathways (Knight, 1972; Schellart and Spekreijse, 1973; van Rossum et al., 2002, 2003). If true, this might create a spike train with slightly irregular intervals ("dithering"), which would desynchronize responses of neighboring ganglion cells, improving the ability of the brain to perceive large objects without greatly reducing its sensitivity.

\section{References}

Ashmore JF, Copenhagen DR (1983) An analysis of transmission from cones to hyperpolarizing bipolar cells in the retina of the turtle. J Physiol (Lond) 340:569-597.

Banks MS, Geisler WS, Bennet PJ (1987) The physical limits of grating visibility. Vision Res 27:1915-1924.

Banks MS, Sekuler AB, Anderson SJ (1991) Peripheral spatial vision: limits imposed by optics, photoreceptors, and receptor pooling. J Opt Soc Am A 8:1775-1787.

Barlow HB (1957) Increment thresholds at low intensities considered as signal/noise discriminations. J Physiol (Lond) 136:469-488.

Barlow HB, Kaushal TP, Hawken M, Parker AJ (1987) Human contrast discrimination and the threshold of cortical neurons. J Opt Soc Am A 4:2366-2371.

Barrett EF, Stevens CF (1972) Quantal independence and uniformity of presynaptic release kinetics at the frog neuromuscular junction. J Physiol (Lond) 227:665-689.

Benison G, Keizer J, Chalupa LM, Robinson DW (2001) Modeling temporal behavior of postnatal cat retinal ganglion cells. J Theor Biol 210:187-199.

Berry MJ, Warland DK, Meister M (1997) The structure and precision of retinal spike trains. Proc Natl Acad Sci USA 94:5411-5416.

Bialek W, Owen WG (1990) Temporal filtering in retinal bipolar cellselements of an optimal computation. Biophys J 58:1227-1233.

Boynton GM, Demb JB, Glover GH, Heeger DJ (1999) Neuronal basis of contrast discrimination. Vision Res 39:257-269.

Chichilnisky EJ, Kalmar RS (2002) Function asymmetries in ON and OFF ganglion cells of primate retina. J Neurosci 22:2737-2747.

Cleland BG, Levick WR, Sanderson KJ (1973) Properties of sustained and transient ganglion cells in the cat retina. J Physiol (Lond) 228:649-680.
Croner LJ, Purpura K, Kaplan E (1993) Response variability in retinal ganglion cells of primates. Proc Natl Acad Sci USA 90:8128-8130.

Dacey DM, Brace SA (1992) Coupled network for parasol but not midget ganglion cells in the primate retina. Vis Neurosci 9:279-290.

Davila KD, Geisler WS (1991) The relative contribution of pre-neural and neural factors to areal summation in the fovea. Vision Res $31: 1369-1380$.

de Ruyter van Steveninck RR, Laughlin SB (1996) The rate of information transfer at graded-potential synapses. Nature 379:642-645.

Demb JB, Zaghloul K, Haarsma L, Sterling P (2001) Bipolar cells contribute to nonlinear spatial summation in the brisk-transient $(\mathrm{Y})$ ganglion cell in mammalian retina. J Neurosci 21:7447-7454.

Derrington AM, Lennie P (1982) The influence of temporal frequency and adaptation level on receptive field organization of retinal ganglion cells in cat. J Physiol (Lond) 333:343-366.

Dhingra NK, Kao YH, Sterling P, Smith RG (2003) Contrast threshold of a brisk-transient ganglion cell in vitro. J Neurophysiol 89:2360-2369.

Diamond JS, Copenhagen DR (1995) The relationship between lightevoked synaptic excitation and spiking behaviour of salamander retinal ganglion cells. J Physiol (Lond) 487:711-725.

Duda RO, Hart PE (1973) Nonparametric techniques. In: Pattern classification and scene analysis, pp 85-129. New York: Wiley.

Enroth-Cugell C, Robson J (1966) The contrast sensitivity of retinal ganglion cells of the cat. J Physiol (Lond) 187:517-552.

Fohlmeister JF, Miller RF (1997) The impulse encoding mechanisms of ganglion cells in the tiger salamander retina. J Neurophysiol 78:1935-1947.

Freed MA (2000) Rate of quantal excitation to a retinal ganglion cell evoked by sensory input. J Neurophysiol 83:2956-2966.

Freed MA, Smith RG, Sterling P (2003) Timing of quantal release from the retinal bipolar terminal is regulated by a feedback circuit. Neuron 38:89-101.

Geisler WS (1989) Sequential ideal-observer analysis of visual discriminations. Psychol Rev 96:267-314.

Geisler WS, Davila KD (1985) Ideal discriminators in spatial vision: twopoint stimuli. J Opt Soc Am A 2:1483-1497.

Geisler WS, Albrecht DG, Salvi RJ, Saunders SS (1991) Discrimination performance of single neurons: rate and temporal-pattern information. J Neurophysiol 66:334-362.

Green DM, Swets JA (1988) Elements of statistical decision theory. In: Signal detection theory and psychophysics, pp 7-29. New York: Wiley.

Haag J, Borst A (1998) Active membrane properties and signal encoding in graded potential neurons. J Neurosci 18:7972-7986.

Ishida AT (1995) Ion channel components of retinal ganglion cells. Prog Retin Eye Res 15:261-280.

Jones JP, Palmer LA (1987) The two-dimensional spatial structure of simple receptive fields in cat striate cortex. J Neurophysiol 58:1187-1211.

Juusola M, French AS (1997) The efficiency of sensory information coding by mechanoreceptor neurons. Neuron 18:959-968.

Kier CK, Buchsbaum G, Sterling P (1995) How retinal microcircuits scale for ganglion cells of different size. J Neurosci 15:7673-7683.

Kim KJ, Rieke F (2003) Slow $\mathrm{Na}^{+}$inactivation and variance adaptation in salamander retinal ganglion cells. J Neurosci 23:1506-1516.

Knight BW (1972) Dynamics of encoding in a population of neurons. J Gen Physiol 59:734-766.

Kretzberg J, Warzecha AK, Egelhaaf M (2001) Neural coding with graded membrane potential changes and spikes. J Comput Neurosci 11:153-164.

Lankheet MJ, Molenaar J, van de Grind WA (1989) Frequency transfer properties of the spike generating mechanism of car retinal ganglion cells. Vision Res 29:1649-1661.

Laughlin SB, de Ruyter van Steveninck RR, Anderson JC (1998) The metabolic cost of neural information. Nat Neurosci 1:36-41.

Levine MW, Zimmerman RP (1991) A model for the response variability of maintained discharge and responses to flashes of light. Biol Cybern 65:469-477.

Linsenmeier RA, Frishman LJ, Jakiela HG, Enroth-Cugell C (1982) Receptive field properties of $\mathrm{X}$ and $\mathrm{Y}$ cells in the cat retina derived from contrast sensitivity measurements. Vision Res 22:1173-1183.

Lipton SA, Tauck DL (1987) Voltage-dependent conductances of solitary ganglion cells dissociated from the rat retina. J Physiol (Lond) 385:361-391.

Nachmias J, Sansbury RV (1974) Grating contrast: discrimination may be better than detection. Vision Res 14:1039-1042. 
Naka KI, Rushton WA (1966) S-potentials from luminosity units in the retina of fish (Cyprinidae). J Physiol (Lond) 185:587-599.

Quick RF (1974) A vector magnitude model of contrast detection. Kybernetik 16:65-67.

Reich DS, Victor JD, Knight BW, Ozaki T, Kaplan E (1977) Response variability and timing precision of neuronal spike trains in vivo. J Neurophysiol 77:2836-2841.

Sakuranaga M, Ando Y, Naka KI (1987) Dynamics of the ganglion cell response in the catfish and frog retina. J Gen Physiol 90:229-259.

Sarpeshkar R, O'Halloran M (2002) Scalable hybrid computation with spikes. Neural Comput 14:2003-2038.

Schellart NAM, Spekreijse H (1973) Origin of the stochastic nature of ganglion cell activity in isolated goldfish retina. Vision Res 13:337-345.

Schneidman E, Freedman B, Segev I (1998) Ion channel stochasticity may be critical in determining the reliability and precision of spike timing. Neural Comp 10:1679-1703.

Shapley R, Victor J (1986) Hypeacuity in cat retinal ganglion cells. Science 231:999-1002.

Simmons PJ (1999) The performance of synapses that convey discrete graded potentials in an insect visual pathway. J Neurosci 19:10584-10594.

Taylor WR, Mittman S, Copenhagen DR (1996) Passive electrical cable properties and synaptic excitation of tiger salamander retinal ganglion cells. Vis Neurosci 13:979-990.

Troy JB, Robson JG (1992) Steady discharges of X and Y retinal ganglion cells of cat under photopic illuminance. Vis Neurosci 9:535-553.

van Rossum MCW, Turrigiano GG, Nelson SB (2002) Fast propagation of firing rates through layered networks of noisy neurons. J Neurosci 22:1956-1966.

van Rossum MCW, O'Brien BJ, Smith RG (2003) Effects of noise on the spike timing precision of retinal ganglion cells. J Neurophysiol 89:2406-2419.

Vaney DI (1991) Many diverse types of retinal neurons show tracer coupling when injected with biocytin or Neurobiotin. Neurosci Lett 125:187-190.

Wang GY, Robinson DW, Chalupa LM (1998) Calcium-activated potassium channels in retinal ganglion cells of the ferret. J Neurophysiol 79:151-158.

White JA, Rubinstein JT, Kay AR (2000) Channel noise in neurons. Trends Neurosci 23:131-137.

Zaghloul KA, Boahen K, Demb JB (2003) Different circuits for ON and OFF retinal ganglion cells cause different contrast sensitivities. J Neurosci 23: 2645-2654. 